\title{
Gross proteinuria is a strong risk predictor for cardiovascular mortality in Brazilian type 2 diabetic patients
}

\author{
C.R.L. Cardoso and G.F. Salles
}

Departamento de Clínica Médica, Hospital Universitário Clementino Fraga Filho, Faculdade de Medicina, Universidade Federal do Rio de Janeiro, Rio de Janeiro, RJ, Brasil

Correspondence to: G.F. Salles, Rua Croton, 72, Jacarepaguá, 22750-240 Rio de Janeiro, RJ, Brasil

Fax: +55-21-2562-2514. E-mail: gilsalles@hucff.ufrj.br

Increased proteinuria is recognized as a risk predictor for all-cause and cardiovascular mortality in diabetic patients; however, no study has evaluated these relationships in Brazilian patients. The aim of this study was to investigate the prognostic value of gross proteinuria for all-cause and cardiovascular mortalities and for cardiovascular morbidity in a cohort study of 471 type 2 diabetic individuals followed for up to 7 years. Several clinical, laboratory and electrocardiographic variables were obtained at baseline. The relative risks for all-cause, cardiovascular and cardiac mortalities and for cardiovascular and cardiac events associated with the presence of overt proteinuria $(>0.5 \mathrm{~g} / 24 \mathrm{~h})$ were assessed by Kaplan-Meier survival curves and by multivariate Cox regression model. During a median follow-up of 57 months (range 2-84 months), 121 patients (25.7\%) died, 44 from cardiovascular and 30 from cardiac causes, and 106 fatal or non-fatal cardiovascular events occurred. Gross proteinuria was an independent risk predictor of all-cause, cardiovascular and cardiac mortalities and of cardiovascular morbidity with adjusted relative risks ranging from 1.96 to 4.38 for the different endpoints. This increased risk remained significant after exclusion of patients with prior cardiovascular disease at baseline from the multivariate analysis. In conclusion, gross proteinuria was a strong predictor of all-cause, cardiovascular and cardiac mortalities and also of cardiovascular morbidity in a Brazilian cohort of type 2 diabetic patients. Intervention studies are necessary to determine whether the reduction of proteinuria can decrease morbidity and mortality of type 2 diabetes in Brazil.

Key words: All-cause mortality; Cardiovascular mortality; Cardiovascular morbidity; Proteinuria; Type 2 diabetes

Both authors have research grants from CNPq (\#301530/2004-1 and \#301547/2005-0).

Received January 17, 2008. Accepted July 14, 2008

\section{Introduction}

Several prospective studies have reported that abnormal proteinuria or albuminuria are predictors of all-cause and cardiovascular mortality in patients with diabetes (18 ), and also in the non-diabetic population (9-11). However, most longitudinal studies on proteinuria and mortality were conducted in European countries (1-5), in the USA $(7,9,11)$ and some in Asia $(6,10)$. Also, there is variability in the extent of the increase in mortality associated with proteinuria among different geographic locations. Thus, it is important to have data from a variety of countries to determine differences in the magnitude of proteinuria- associated risks of specific causes of death and incidence of non-fatal events. In particular, the role of proteinuria as a predictive marker of mortality is not known in some diabetic populations. No Brazilian study has described the association between proteinuria and total mortality and cardiovascular morbidity and mortality. We have previously reported the risk markers of all-cause mortality and of stroke in this cohort and proteinuria was among the independent predictors $(12,13)$.

Hence, the aim of this study was to investigate the relationship between gross proteinuria and total all-cause, cardiovascular and cardiac mortalities and cardiovascular morbidity in a Brazilian multi-ethnic cohort prospective study. 


\section{Patients and Methods}

\section{Study patients and baseline procedures}

Study patients have been described elsewhere $(14,15)$. Briefly, all adult diabetic outpatients, diagnosed according to 1985 WHO criteria, who had standard EKGs recorded from July 1994 to June 1996 were enrolled consecutively in the study. Exclusion criteria were type 1 diabetes, unstable angina or recent (less than 4 months) acute myocardial infarction, serum potassium or calcium electrolyte abnormalities, use of any antiarrhythmic drug (except $\beta$ blockers when used for hypertension or coronary artery disease), chronic renal failure undergoing dialysis and non-sinusal rhythms on EKG. After applying these exclusion criteria, a total of 471 type 2 diabetic patients composed the cohort. The study protocol complied with the 1975 Declaration of Helsinki, was approved by the local Ethics Committee and all patients gave written informed consent.

The baseline procedures and criteria for diagnosing clinical variables have been described previously $(14,15)$. In brief, all subjects were submitted to a complete clinical examination, with special attention to signs and symptoms of cardiovascular diseases and to diabetic degenerative complications. Laboratory evaluation included fasting plasma glucose, creatinine, triglycerides, total and HDL cholesterol, and 24-h proteinuria, by automated methods. The diagnosis of gross proteinuria, consistent with clinical diabetic nephropathy, needed at least 3 consecutive measurements greater than $0.5 \mathrm{~g} / 24 \mathrm{~h}$. Patients with previous persistent overt proteinuria $(>0.5 \mathrm{~g} / 24 \mathrm{~h})$ were considered as clinical nephropathy independent of present level of proteinuria. No anthropometric measures were obtained. Arterial hypertension was diagnosed if mean systolic blood pressure $\geq 140 \mathrm{mmHg}$ or diastolic blood pressure $\geq 90$ $\mathrm{mmHg}$ or if anti-hypertensive drugs had been prescribed. Mean values of all blood pressure measurements and laboratory examinations performed in the first year of follow-up were recorded.

\section{Follow-up and endpoints}

The patients were evaluated regularly at least two times a year until June 2001. Those who failed to return to the hospital clinic were contacted annually to determine health status. Causes of death during the follow-up period were ascertained from medical records, death certificates and interviews with attending physicians and families, by a standard questionnaire reviewed by an independent observer. Causes of death were coded according to the International Classification of Disease. The primary endpoints were allcause, cardiovascular and cardiac mortalities. Cardiac mortality was defined as death from acute myocardial infarction, progressive heart failure or sudden cardiac death, defined as death occurring instantaneously or within $1 \mathrm{~h}$ after the onset of symptoms (16). Cardiovascular mortality was defined as death from any cardiac, cerebrovascular, aortic or peripheral arterial disease. Total, fatal or non-fatal, cardiovascular and cardiac events were also primary endpoints. We considered as cardiac events all cardiac deaths plus non-fatal myocardial infarctions and any myocardial revascularization procedures (either surgical or not), and as cardiovascular events all cardiac events and cardiovascular deaths plus strokes, amputations above the ankle and lower limb revascularization procedures. The observation period for each patient was the number of months from the date of entry in the study to the date of death or June 30, 2001. Fortythree patients $(9.1 \%)$ were lost from follow-up and were considered censored observations at the date of their last hospital visit.

\section{Statistical analysis}

All statistics were performed using the SPSS statistical package version 13.0. Continuous data were reported as means and standard deviations. Comparisons of baseline characteristics between the subgroups with and without gross proteinuria were performed by the unpaired $t$-test for continuous variables with normal distribution, by the MannWhitney test for continuous asymmetrically distributed variables and by the chi-square test for categorical data. Kaplan Meyer estimation of mortality curves (compared by log-rank test) and univariate and multivariate proportional hazards Cox models were used for survival analysis. Cox regression models were fitted in a forward stepwise procedure for each primary endpoint. First, it was adjusted for age and sex and then for all other covariates that could potentially impact the associations between gross proteinuria and outcome (age, gender, diabetes duration, presence of arterial hypertension and any cardiovascular disease at baseline, mean systolic and diastolic blood pressures, anti-hypertensive and diabetic treatment, lipid profile and mean fasting glycemia). In addition, regression analysis was repeated for all endpoints after excluding patients with any cardiovascular disease at baseline. Assumptions of the proportional hazards models and interactions were also tested (17) and no violation was observed. Results are reported as adjusted relative risks (RRs) with their 95\% confidence intervals. A two-tailed $\mathrm{P}$ value $<0.05$ was considered to be statistically significant.

\section{Results}

Baseline characteristics

Mean proteinuria ranged from 0.01 to $10.00 \mathrm{~g} / 24 \mathrm{~h}$ (median: 0.18 , interquartile range: $0.06-0.46 \mathrm{~g} / 24 \mathrm{~h}$ ). One 
hundred and six patients $(22.5 \%)$ presented gross proteinuria. Baseline characteristics of all patients and of those with and without overt proteinuria are reported in Table 1. Patients with gross proteinuria were more frequently males, had a longer duration of diabetes, increased prevalence of arterial hypertension, cardiovascular diseases and diabetic retinopathy. They also had higher systolic and diastolic blood pressures and heart rate, used angiotensin-converting enzyme inhibitor drugs more frequently and had higher serum triglycerides than those without clinical nephropathy.

Follow-up endpoints and survival analysis

During a median follow-up of 57 months (range 2-84 months), there were 121 all-cause deaths, 44 from cardiovascular and 30 from cardiac causes. There were 106 total fatal or non-fatal cardiovascular events, 40 strokes, and 55 cardiac events. Results of multivariate Cox regression analysis of all patients are reported in Table 2. After adjustment for age and gender, the presence of gross proteinuria was independently associated with all-cause, cardiovascular and cardiac mortalities and also with total, fatal or non-fatal, cardiovascular, cardiac and cerebrovascular events. After adjusting for all covariates, the presence of overt proteinuria remained a significant predictor of all endpoints except for the occurrence of cardiac events. RRs after full adjustment ranged from 1.6 for cardiac events up to 4.4 for cardiac and cardiovascular mortalities. Table 3 shows the same multivariate analysis after excluding 130 pa-
Table 1. Baseline characteristics of all patients with and without gross proteinuria.

\begin{tabular}{|c|c|c|c|}
\hline Characteristics & $\begin{array}{l}\text { All patients } \\
(\mathrm{N}=471)\end{array}$ & $\begin{array}{l}\text { Patients with } \\
\text { proteinuria } \\
>0.5 \mathrm{~g} / 24 \mathrm{~h} \\
(\mathrm{~N}=106)\end{array}$ & $\begin{array}{l}\text { Patients with } \\
\text { proteinuria } \\
\leq 0.5 \mathrm{~g} / 24 \mathrm{~h} \\
(\mathrm{~N}=365)\end{array}$ \\
\hline Gender (male) & $161(34.2 \%)$ & $51(48.1 \%)^{+}$ & $110(30.1 \%)$ \\
\hline Age (years) & $60.5 \pm 11.1$ & $58.5 \pm 10.1^{*}$ & $61.0 \pm 11.3$ \\
\hline Diabetes duration (years) & $9.3 \pm 7.3$ & $10.1 \pm 7.7^{\#}$ & $9.0 \pm 7.3$ \\
\hline Retinopathy & $95(20.2 \%)$ & $33(31.3 \%)^{+}$ & $62(17.0 \%)$ \\
\hline Neuropathy & $68(14.4 \%)$ & $18(17.0 \%)$ & $50(13.7 \%)$ \\
\hline Arterial hypertension & $271(57.5 \%)$ & $70(66.0 \%)^{\star}$ & $201(55.1 \%)$ \\
\hline Heart failure & $20(4.2 \%)$ & $11(10.4 \%)^{+}$ & $9(2.5 \%)$ \\
\hline Coronary heart disease & $56(11.9 \%)$ & $17(16.0 \%)$ & 39 (10.7\%) \\
\hline Cerebrovascular disease & $26(5.5 \%)$ & $3(2.8 \%)$ & $23(6.3 \%)$ \\
\hline Peripheral vascular disease & $43(9.1 \%)$ & $14(13.2 \%)$ & $29(7.9 \%)$ \\
\hline $\mathrm{SBP}(\mathrm{mmHg})$ & $142.8 \pm 19.8$ & $146.1 \pm 19.8^{+}$ & $141.9 \pm 19.7$ \\
\hline $\mathrm{DBP}(\mathrm{mmHg})$ & $83.3 \pm 10.5$ & $85.4 \pm 10.0^{+}$ & $82.7 \pm 10.6$ \\
\hline Heart rate (bpm) & $78.0 \pm 14.6$ & $83.0 \pm 14.6^{\#}$ & $76.6 \pm 14.5$ \\
\hline \multicolumn{4}{|l|}{ Diabetes treatment } \\
\hline Sulfonylureas & $274(58.2 \%)$ & $63(59.4 \%)$ & $211(57.8 \%)$ \\
\hline Insulin & $130(27.6 \%)$ & $27(25.5 \%)$ & $103(28.2 \%)$ \\
\hline \multicolumn{4}{|l|}{ Anti-hypertensive treatment } \\
\hline B blockers & $36(7.6 \%)$ & $8(7.5 \%)$ & $28(7.7 \%)$ \\
\hline Calcium channel blockers & $94(20.0 \%)$ & $24(22.6 \%)$ & $70(19.2 \%)$ \\
\hline Diuretics & $111(23.6 \%)$ & $24(22.6 \%)$ & 79 (21.6\%) \\
\hline ACE inhibitors & $135(28.7 \%)$ & $53(50.0 \%)^{\#}$ & 82 (22.5\%) \\
\hline Fasting glycemia (mmol/L) & $10.7 \pm 4.0$ & $10.5 \pm 4.4$ & $10.8 \pm 3.9$ \\
\hline Triglycerides (mmol/L) & $1.92 \pm 1.12$ & $2.23 \pm 1.51^{*}$ & $1.84 \pm 0.96$ \\
\hline Total cholesterol (mmol/L) & $5.38 \pm 1.26$ & $5.67 \pm 1.54$ & $5.30 \pm 1.16$ \\
\hline HDL cholesterol (mmol/L) & $1.07 \pm 0.27$ & $1.04 \pm 0.32$ & $1.08 \pm 0.26$ \\
\hline
\end{tabular}

Data are reported as absolute numbers with percent in parentheses or means $\pm \mathrm{SD}$. SBP = systolic blood pressure; $\mathrm{DBP}=$ diastolic blood pressure; $\mathrm{ACE}=$ angiotensin-converting enzyme; $\mathrm{HDL}=$ high-density lipoprotein .

${ }^{*} \mathrm{P}<0.05,{ }^{+} \mathrm{P}<0.01,{ }^{\#} \mathrm{P}<0.001$ comparisons between patients with and without abnormal proteinuria (unpaired $t$-test, Mann-Whitney and $\chi^{2}$ tests).

Table 2. Adjusted relative risks associated with the presence of gross proteinuria for each endpoint of all 471 diabetic patients.

\begin{tabular}{|c|c|c|c|c|c|c|}
\hline & \multicolumn{3}{|c|}{ Model A } & \multicolumn{3}{|c|}{ Model B } \\
\hline & $\mathrm{RR}$ & $95 \% \mathrm{Cl}$ & $\mathrm{P}$ & $\mathrm{RR}$ & $95 \% \mathrm{Cl}$ & $P$ \\
\hline All-cause mortality $(\mathrm{N}=121)$ & 2.61 & $1.78-3.83$ & $<0.001$ & 2.55 & $1.68-3.87$ & $<0.001$ \\
\hline Cardiovascular mortality $(\mathrm{N}=44)$ & 4.59 & $2.50-8.42$ & $<0.001$ & 4.38 & $2.25-8.52$ & $<0.001$ \\
\hline Cardiac mortality $(\mathrm{N}=30)$ & 4.51 & $2.16-9.40$ & $<0.001$ & 4.37 & $1.94-9.85$ & $<0.001$ \\
\hline Total cardiac events $(\mathrm{N}=55)$ & 2.07 & $1.15-3.72$ & 0.016 & 1.57 & $0.81-2.98$ & 0.18 \\
\hline Stroke $(N=40)$ & 2.63 & $1.34-5.16$ & 0.005 & 2.96 & $1.41-6.18$ & 0.004 \\
\hline Total cardiovascular events $(\mathrm{N}=106)$ & 2.31 & $1.53-3.50$ & $<0.001$ & 1.96 & $1.23-3.12$ & 0.005 \\
\hline
\end{tabular}

Adjusted relative risk was calculated with the Cox regression model. $\mathrm{RR}=$ relative risk; $\mathrm{N}=$ number of events. Model $\mathrm{A}$ : adjusted for age and gender. Model B: adjusted for age, gender, diabetes duration, arterial hypertension, cardiovascular disease and retinopathy at baseline, hypertension and diabetes treatment, systolic and diastolic blood pressures, heart rate, serum total and HDL cholesterol, triglycerides and fasting glycemia. 
Table 3. Adjusted relative risks associated with the presence of gross proteinuria for each endpoint of 341 diabetic patients without prior cardiovascular disease.

\begin{tabular}{|c|c|c|c|c|c|c|}
\hline & \multicolumn{3}{|c|}{ Model A } & \multicolumn{3}{|c|}{ Model B } \\
\hline & $\mathrm{RR}$ & $95 \% \mathrm{Cl}$ & $\mathrm{P}$ & $\mathrm{RR}$ & $95 \% \mathrm{Cl}$ & $\mathrm{P}$ \\
\hline All-cause mortality ( $N=69)$ & 2.17 & $1.29-3.65$ & 0.003 & 2.41 & $1.36-4.27$ & 0.003 \\
\hline Cardiovascular mortality $(\mathrm{N}=24)$ & 4.73 & $2.10-10.67$ & $<0.001$ & 4.85 & 1.97-11.93 & 0.001 \\
\hline Cardiac mortality $(\mathrm{N}=15)$ & 3.58 & $1.27-10.12$ & 0.016 & 3.59 & $1.14-11.23$ & 0.028 \\
\hline Total cardiac events $(\mathrm{N}=28)$ & 1.68 & $0.71-3.97$ & 0.24 & 1.77 & $0.68-4.60$ & 0.24 \\
\hline Stroke $(N=26)$ & 2.12 & $0.89-5.08$ & 0.09 & 2.34 & $0.90-6.11$ & 0.08 \\
\hline Total cardiovascular events $(\mathrm{N}=57)$ & 1.99 & $1.11-3.56$ & 0.021 & 2.12 & $1.10-4.08$ & 0.024 \\
\hline
\end{tabular}

Adjusted relative risk was calculated with the Cox regression model. $\mathrm{RR}=$ relative risk; $\mathrm{N}=$ number of events. Model $\mathrm{A}$ : adjusted for age and gender. Model B: adjusted for age, gender, diabetes duration, arterial hypertension and retinopathy at baseline, hypertension and diabetes treatment, systolic and diastolic blood pressures, heart rate, serum total and HDL cholesterol, triglycerides and fasting glycemia.
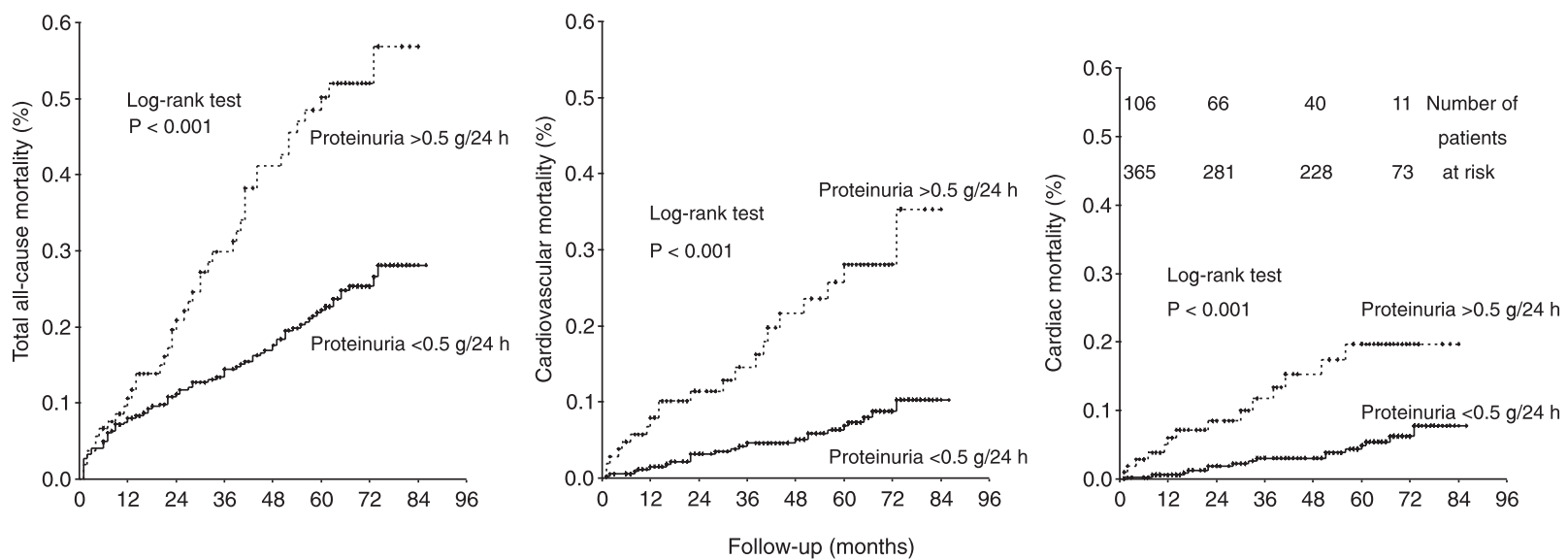

Figure 1. Kaplan-Meier estimation of all-cause, cardiovascular and cardiac mortality curves in patients grouped according to the presence of gross proteinuria in all 471 diabetic patients. The number of patients at risk at each 2-year period is shown in the right panel.

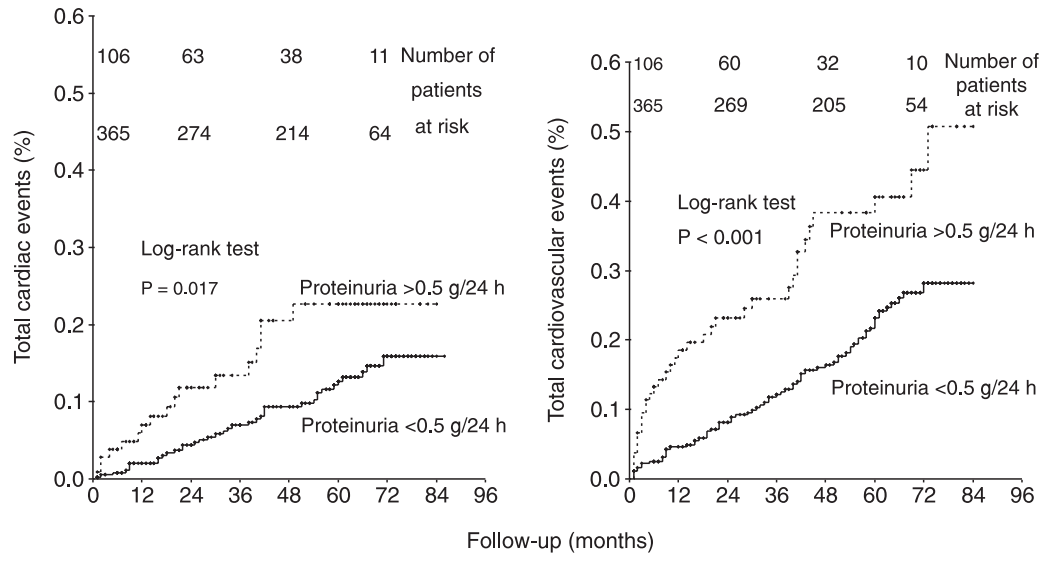

Figure 2. Kaplan-Meier estimation of incident total fatal or non-fatal cardiovascular and cardiac event curves in patients grouped according to the presence of gross proteinuria in all 471 diabetic patients. The number of patients at risk at each 2-year period is shown in each panel. 
tients with pre-existent cardiovascular disease at baseline. In patients without any cardiovascular disease, the presence of gross proteinuria remained a predictor of poor prognosis for all endpoints investigated, except for total cardiac events and stroke. In the fully adjusted models, the RRs ranged from 1.8 for cardiac events to nearly 5.0 for cardiovascular mortality.

Figures 1 and 2 show Kaplan-Meier curves for the 5 primary endpoints. The presence of gross proteinuria distinguishes a subgroup of diabetic patients with significantly worse prognosis than those with lower proteinuria levels. Figure 3 shows the Kaplan-Meier curves for the mortality endpoints excluding the patients with prior cardiovascular disease. Similarly, the presence of overt proteinuria was associated with greater mortality, even in those diabetic patients without any cardiovascular disease at baseline.

\section{Discussion}

In this prospective cohort study with up to 7 years of follow-up in a Brazilian type 2 diabetic population, the presence of persistent gross proteinuria, defined as a mean urinary protein excretion rate greater than $0.5 \mathrm{~g} / 24 \mathrm{~h}$, predicted not only increased risk of all-cause, cardiovascular and cardiac mortalities, but also of incident total, fatal or non-fatal, cardiovascular events, with relative risks varying from nearly twice for total cardiovascular events up to 4.5-fold for cardiovascular mortality compared with the reference group without gross proteinuria at baseline. These increased risks persisted independent after adjusting for other important cardiovascular risk factors, such as presence of arterial hypertension and cardiovascular dis- ease at baseline, blood pressure levels and serum lipid profile. They were also observed in the subgroup of diabetic patients without cardiovascular disease at entry into the study.

These results agree with previous studies (1,4,6-8, $18,19)$. Overt proteinuria or macroalbuminuria are considered to be, in general, good biomarkers of subsequent mortality $(9,10,20)$. Various studies have shown a better survival in non-proteinuric type 2 diabetic patients. Nevertheless, most of these studies are based on simple qualitative dipstick or precipitation screening tests $(1,6,7,9-11,18)$ or on urinary protein concentration from a morning spot (19). In the present study, the measurement of 24-h urinary protein excretion was assessed on at least three different occasions, whenever possible. Certainly, the method performed in this study, decreased random proteinuria variation and increased the strength of the associations found between gross proteinuria and morbi-mortality.

This is the first prospective study performed in a multiethnic Brazilian population, confirming the predictive role of proteinuria in type 2 diabetic patients. However, absence of information on urinary albumin excretion rate is a real limitation of this study, preventing the evaluation of the prognostic value of lower levels of proteinuria and microalbuminuria. Even though some studies have reported similar predictive roles of both gross proteinuria and microalbuminuria for cardiovascular death $(7,8)$, and other investigations have observed an even stronger association of overt proteinuria than microalbuminuria with cardiovascular mortality $(4,21)$, data on albuminuria would certainly improve this study.

Many explanations have been proposed to account for
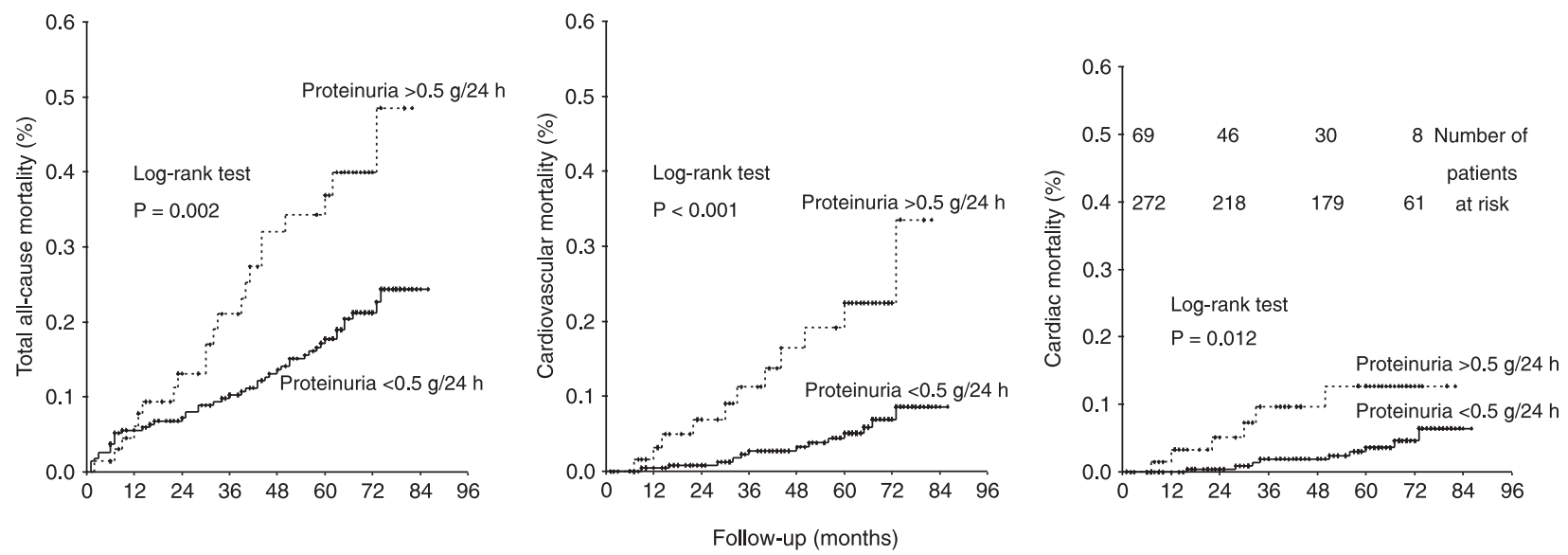

Figure 3. Kaplan-Meier estimation of all-cause, cardiovascular and cardiac mortality curves in patients grouped according to the presence of gross proteinuria in 341 patients without cardiovascular disease at baseline. The number of patients at risk at each 2year period is shown in the right panel. 
the increased cardiovascular morbidity and mortality in type 2 diabetes related to abnormal urinary protein excretion. The exact mechanism by which increased urinary protein excretion is associated with increased cardiovascular risk is unclear; but it is probably multifactorial (22). One possible mechanism may be excessive protein reabsorption in the proximal renal tubule, causing increased synthesis of vasoactive and inflammatory substances (23). This inflammatory response may elicit local and possibly systemic endothelial dysfunction. Endothelial dysfunction occurs precociously in atherosclerosis and modulates vascular permeability. Increased vascular permeability related to proteinuria may reflect systemic endothelial dysfunction and chronic inflammation. On the other hand, the presence of proteinuria may represent a surrogate marker of diffuse systemic vascular disease (24), reflecting the increased transvascular leak of a variety of proteins, including lipoproteins (22-24), that leads to atherosclerotic vascular disease. Although a lot of work has been done to clarify these complex interactions, a more comprehensive understanding is still necessary.

The present study has some limitations that include the fact that albuminuria and other potentially important prognostic variables, such as glycated hemoglobin, smoking status and body mass index were not included in the analysis because they were not available. Smoking status was considered to be non-reliable data for this cohort. It has been reported as a mortality risk factor in some investigations $(1,21,25)$, but not in others $(4,6,8,26)$. Information on metabolic control provided by $\mathrm{HbA}_{1 \mathrm{c}}$ levels probably was, at least partially, provided by mean fasting glycemia

\section{References}

1. Morrish NJ, Stevens LK, Fuller JH, Jarrett RJ, Keen H. Risk factors for macrovascular disease in diabetes mellitus: the London follow-up to the WHO Multinational Study of Vascular Disease in Diabetics. Diabetologia 1991; 34: 590-594.

2. Neil A, Hawkins M, Potok M, Thorogood M, Cohen D, Mann J. A prospective population-based study of microalbuminuria as a predictor of mortality in NIDDM. Diabetes Care 1993; 16: 996-1003.

3. MacLeod JM, Lutale J, Marshall SM. Albumin excretion and vascular deaths in NIDDM. Diabetologia 1995; 38: 610-616.

4. Gall MA, Borch-Johnsen K, Hougaard P, Nielsen FS, Parving $\mathrm{HH}$. Albuminuria and poor glycemic control predict mortality in NIDDM. Diabetes 1995; 44: 1303-1309.

5. Hanninen J, Takala J, Keinanen-Kiukaanniemi S. Albuminuria and other risk factors for mortality in patients with noninsulin-dependent diabetes mellitus aged under 65 years: a population-based prospective 5-year study. Diabetes Res Clin Pract 1999; 43: 121-126. levels obtained during the first year of follow-up. Furthermore, no investigation for silent myocardial ischemia by provocative tests was performed, since the diagnosis of pre-existent coronary heart disease was made by clinical and electrocardiographic criteria. Thus, due to the absence of these covariates, the possibility of an incomplete statistical adjustment cannot be ruled out. However, the relationships between gross proteinuria and the various outcomes found in this study are similar to other studies. Therefore, we believe that it is improbable that the absence of these variables could have significantly affected our results, given the strength and consistency of the associations we detected. Another possible limitation was the relatively small number of endpoints observed, especially in the analysis excluding the patients with pre-existent cardiovascular disease at baseline. This may have affected the accuracy of risk estimates for this particular survival analysis. Finally, another limitation was the selection of patients for this study. Since it was not a populationbased, but a hospital-based cohort, the diabetic patients of this investigation may not be representative of the general Brazilian type 2 diabetic population.

This prospective study provides evidence that gross proteinuria is a strong independent predictor of all-cause, cardiovascular and cardiac mortalities and of cardiovascular morbidity in Brazilian type 2 diabetic patients. These increased risks are also present in patients without any cardiovascular disease at baseline. Multi-interventional studies are needed to try to reduce mortality and morbidity related to the degenerative complications of diabetes in Brazil.
6. Chen KT, Chen CJ, Fuh MM, Narayan KM. Causes of death and associated factors among patients with non-insulindependent diabetes mellitus in Taipei, Taiwan. Diabetes Res Clin Pract 1999; 43: 101-109.

7. Valmadrid CT, Klein R, Moss SE, Klein BE. The risk of cardiovascular disease mortality associated with microalbuminuria and gross proteinuria in persons with older-onset diabetes mellitus. Arch Intern Med 2000; 160: 1093-1100.

8. Casiglia E, Zanette G, Mazza A, Donadon V, Donada C, Pizziol A, et al. Cardiovascular mortality in non-insulin-dependent diabetes mellitus. A controlled study among 683 diabetics and 683 age- and sex-matched normal subjects. Eur J Epidemiol 2000; 16: 677-684.

9. Grimm RH Jr, Svendsen KH, Kasiske B, Keane WF, Wahi MM. Proteinuria is a risk factor for mortality over 10 years of follow-up. MRFIT Research Group. Multiple Risk Factor Intervention Trial. Kidney Int Suppl 1997; 63: S10-S14.

10. Tanihara S, Hayakawa T, Oki I, Nakamura Y, Sakata K, 
Okayama A, et al. Proteinuria is a prognostic marker for cardiovascular mortality: NIPPON DATA 80, 1980-1999. J Epidemiol 2005; 15: 146-153.

11. Madison JR, Spies C, Schatz IJ, Masaki K, Chen R, Yano K, et al. Proteinuria and risk for stroke and coronary heart disease during 27 years of follow-up: the Honolulu Heart Program. Arch Intern Med 2006; 166: 884-889.

12. Cardoso CR, Salles GF, Deccache W. QTc interval prolongation is a predictor of future strokes in patients with type 2 diabetes mellitus. Stroke 2003; 34: 2187-2194.

13. Salles GF, Bloch KV, Cardoso CR. Mortality and predictors of mortality in a cohort of Brazilian type 2 diabetic patients. Diabetes Care 2004; 27: 1299-1305.

14. Cardoso C, Salles G, Bloch K, Deccache W, Siqueira-Filho AG. Clinical determinants of increased QT dispersion in patients with diabetes mellitus. Int J Cardiol 2001; 79: 253262.

15. Cardoso CR, Salles GF, Deccache W. Prognostic value of QT interval parameters in type 2 diabetes mellitus: results of a long-term follow-up prospective study. J Diabetes Complications 2003; 17: 169-178.

16. Myerburg RJ, Kessler KM, Castellanos A. Sudden cardiac death. Structure, function, and time-dependence of risk. Circulation 1992; 85 (Suppl): I-2-I-10.

17. Concato J, Feinstein AR, Holford TR. The risk of determining risk with multivariable models. Ann Intern Med 1993; 118: $201-210$

18. Sasaki A, Uehara M, Horiuchi N, Hasegawa K, Shimizu T. A 15-year follow-up study of patients with non-insulin-dependent diabetes mellitus (NIDDM) in Osaka, Japan. Factors predictive of the prognosis of diabetic patients. Diabetes
Res Clin Pract 1997; 36: 41-47.

19. Miettinen $\mathrm{H}$, Haffner SM, Lehto S, Ronnemaa T, Pyorala K, Laakso M. Proteinuria predicts stroke and other atherosclerotic vascular disease events in nondiabetic and non-insulin-dependent diabetic subjects. Stroke 1996; 27: 20332039.

20. Damsgaard EM, Froland A, Jorgensen OD, Mogensen CE. Eight to nine year mortality in known non-insulin dependent diabetics and controls. Kidney Int 1992; 41: 731-735.

21. Rossing $\mathrm{P}$, Hougaard $\mathrm{P}$, Borch-Johnsen $\mathrm{K}$, Parving $\mathrm{HH}$. Predictors of mortality in insulin dependent diabetes: 10 year observational follow up study. BMJ 1996; 313: 779784.

22. Paisley KE, Beaman M, Tooke JE, Mohamed-Ali V, Lowe GD, Shore AC. Endothelial dysfunction and inflammation in asymptomatic proteinuria. Kidney Int 2003; 63: 624-633.

23. Remuzzi G, Ruggenenti P, Perico N. Chronic renal diseases: renoprotective benefits of renin-angiotensin system inhibition. Ann Intern Med 2002; 136: 604-615.

24. Jensen JS. Renal and systemic transvascular albumin leakage in severe atherosclerosis. Arterioscler Thromb Vasc Biol 1995; 15: 1324-1329.

25. Wei M, Gaskill SP, Haffner SM, Stern MP. Effects of diabetes and level of glycemia on all-cause and cardiovascular mortality. The San Antonio Heart Study. Diabetes Care 1998; 21: 1167-1172.

26. Ostgren CJ, Lindblad U, Melander A, Rastam L. Survival in patients with type 2 diabetes in a Swedish community: Skaraborg Hypertension and Diabetes Project. Diabetes Care 2002; 25: 1297-1302. 